\title{
Indigenismos en el discurso médico de Guatemala del siglo XVIII: El caso de la Instrucción sobre el modo de practicar la inoculación de las viruelas de José Felipe Flores
}

\section{Indigenisms in the Medical Discourse of Guatemala in the 18th Century: \\ The case of Instrucción sobre el modo de practicar la inoculación \\ de las viruelas, by José Felipe Flores}

Mara Yolanda Montero Lazcano [mara.monterolazcano@gmail.com]

Universidad Autónoma de Querétaro, México

\section{RESUMEN}

Este trabajo versa sobre los indigenismos presentes en el discurso médico de Guatemala del siglo XVIII registrados en la Instrucción sobre el modo de practicar la inoculación de las viruelas del Dr. José Felipe Flores. El análisis se hace a partir de la identificación y clasificación de las voces indígenas, así como de la revisión de sus contextos de aparición para determinar el grado de integración y contacto con el español, dando como resultado una estrecha relación entre el campo de la medicina y el de la alimentación, común en la tradición mesoamericana. El propósito final de esta investigación es la aportación de datos que permitan contribuir al estudio del léxico médico centroamericano.

\section{Palabras Clave}

Historia del español de América; español de Guatemala; siglo XVIII; indigenismos; medicina; alimentación

\begin{abstract}
This paper talks about the indigenisms identified in the medical discourse of Guatemala during the 18th century recorded in the Instrucción sobre el modo de practicar la inoculación de las viruelas by Dr. José Felipe Flores. The analysis involves the identification and classification of indigenous voices, as well as the review of their contexts to determine their level of integration and contact with the Spanish language. As a result, a close relationship is found in fields such as medicine and food, a tendency commonly found on the Mesoamerican tradition. The final purpose of this research is to contribute with useful data to the study of the medical lexicon of Central America.
\end{abstract}

\section{KEYWORDS}

History of Latin American Spanish; Guatemala Spanish; 18th century; indigenisms; medicine; food 


\section{Introducción}

El léxico de la medicina durante el siglo XVIII es un tema que, independientemente de la zona geográfica en donde se origine el texto, resulta interesante para la investigación lingüística; no obstante, lugares como España han captado mayor atención para el desarrollo de estos estudios (Gómez de Enterría 2012a, 2012b, 2013; Gómez de Enterría y Gallardo 2010; Gutiérrez Rodilla 1993, 1994-5, 1996, entre otros).

En el caso del continente americano, recientemente ha crecido el número de trabajos publicados sobre este tema (Parcero Torre 2011; Gómez de Enterría 2014; Ramírez Luengo 2014, 2015, 2019), aunque su estudio sigue teniendo importantes vacíos como es el caso del aporte léxico proveniente de aquellas lenguas originarias que, al entrar en contacto con el español, reciben el nombre de indigenismos (Mejías 1980; Polo Cano 2005; García Frazier 2006; Ramírez Luengo 2017, en prensa; entre otros). Con esto en mente, la presente investigación se centra en el análisis de las voces indígenas que aparecen en un texto médico centroamericano de la segunda mitad del siglo XVIII, cuyo nombre original es Instrucción sobre el modo de practicar la inoculación de las viruelas, y método para curar esta enfermedad, acomodado a la naturaleza, y modo de vivir de los indios, del Reino de Guatemala ${ }^{1}$, escrito por el Dr. José Felipe Flores y publicado en la actual ciudad de Guatemala en 1794.

Para el análisis de los indigenismos, es necesario hacer una lectura cuidadosa del texto y así poder listar todas las voces de origen autóctono localizadas. Posteriormente se clasifican por origen etimológico y campo semántico buscando en ambos factores las tendencias mayoritarias y, por último, a partir de una revisión de diversas fuentes lexicográficas y corpus lingüísticos, se determina cuáles de dichas voces muestran en el texto analizado su primera datación, tanto general como en Centroamérica, así como su grado de incorporación en el español de la época.

\section{Datos generales del autor y de la obra}

De acuerdo con autores como Garzón y Rincón (2014) y Barona Vilar (2019) José Felipe Flores nace en Ciudad Real de Chiapas en el año de 1751. Sus primeros estudios son sobre Latinidades en el Seminario Tridentino en su ciudad natal y posteriormente, para el año de 1773, se gradúa como Bachiller en Medicina de la Universidad de San Carlos, en Santiago de los Caballeros. Para 1774, debido a su gran capacidad, se convierte en el director del hospital de San Juan de Dios, y en 1778 intenta concursar por la cátedra de Prima Medicinae, con el tema Ventajas de la inoculación de las viruelas y la necesidad de establecer esta operación en este reino para precaver los estragos de esta funesta enfermedad; sin embargo, en ese momento pierde la oposición. Un año más tarde, al mismo tiempo que obtiene su título de Licenciado en Medicina, Guatemala se ve afectada por una epidemia de viruela ${ }^{2}$ que cobra la vida de más de diez mil niños y jóvenes indios, por lo que José Felipe Flores tiene la oportunidad de poner en práctica la técnica de inoculación, que posterior-

1 Las voces, ejemplos y fragmentos tomados del texto original son adaptados a la escritura del español mexicano actual.

2 Para más información acerca de esta cuestión, conviene revisar a Lovell (1988) quien hace una revisión sobre la mortandad indígena a causa de la viruela en la Sierra de los Cuchumatanes durante este periodo. 
mente es registrada por él mismo en el texto que se analiza en este trabajo, ayudando a que la tasa de mortandad descienda hasta uno por cada doscientos habitantes (Garzón y Rincón 2014: 25).

La Instrucción sobre el modo de practicar la inoculación de las viruelas es un texto breve que, junto con otro editado años antes sobre el cáncer o "el mal del cancro", forman las dos únicas publicaciones conocidas de José Felipe Flores. La Instrucción cuenta con diecisiete páginas, en las primeras diez se puede observar cada una de las indicaciones que el doctor sugiere se lleven a cabo para practicar la inoculación y así prevenir el contagio de la viruela, estas son: 1 . Lo que se ha de hacer antes de practicar la inoculación; 2. Modo de practicar la inoculación; 3. Método para curar a los inoculados; 4. Método para curar las viruelas naturales; 5 . Lo que se deberá practicar para impedir que la epidemia de las viruelas pase de los pueblos apestados a los que aún no lo están (Flores 1794).

Respecto al resto del documento, se debe señalar que se trata de memoriales del autor, así como de un certificado donde se autoriza sacar dinero de las cajas de las comunidades para efectos de la inoculación. Por lo tanto, no cabe duda de que el texto tiene un propósito específico: instruir a las respectivas instancias de gobierno para evitar la propagación de la viruela a partir de una adecuada inoculación; haciéndose evidente el carácter divulgativo de la obra.

\section{Indigenismos en el discurso médico}

Como es sabido, el encuentro de dos mundos, con dos realidades diferentes, motiva el enriquecimiento de la cultura occidental no solo a nivel de conocimientos de distinta naturaleza, sino también a nivel de vocabulario. Uno de los campos con mayor ampliación léxica es sin duda el referente a los tratamientos de índole medicinal, ya que el contacto entre españoles y pueblos originarios de América conlleva una apropiación de métodos y tratamientos para curar diversas enfermedades a partir de las tradiciones indoamericanas. De acuerdo con Ramírez Luengo (en prensa), "la necesidad de nombrar productos propios de las tierras americanas que no tienen correlato en Europa obliga a los españoles a emplear en numerosas ocasiones lo que se ha denominado la estrategia léxica de incorporación, que se entiende como la adaptación de unidades léxicas originarias de otros sistemas lingüísticos para designar estos nuevos referentes".

En coincidencia con lo que se acaba de señalar, dentro del texto sobre la inoculación de las viruelas del Dr. José Felipe Flores se puede observar un total de doce indigenismos ${ }^{3}$, a saber: achiote, atole, cacao, camote, chay, chicha, chile, chocolate, güisquil, jícara, nixtamal y súchilt, todos en las primeras páginas de la instrucción. Las apariciones de voces indígenas parecen en principio pocas, pero a este respecto conviene recordar la brevedad del documento y la escasa presencia de estos vocablos en textos centroamericanos dieciochescos (San Martín Gómez 2019: 124; Ramírez Luengo, Aquino Melchor y Ramírez Vázquez 2017: 14), por lo que resultan suficientes para realizar un análisis de interés que enriquezca la información acerca de su empleo en tipologías y zonas geográficas poco analizadas como son los textos médicos y Guatemala. A continuación, se iniciará

3 Dentro del documento también aparecen algunos topónimos tomados de las lenguas originarias de la zona, pero no han sido considerados en esta investigación al tener otro método de trabajo para su análisis.

4 Todas las voces indígenas fueron actualizadas a las normas ortográficas actuales del español. 
la clasificación de los indigenismos localizados a partir de los factores de estudio ya mencionados, es decir, su origen etimológico y el campo léxico al que pertenecen, así como su grado de incorporación en la lengua española y sus primeras dataciones.

\subsection{Origen etimológico}

El origen etimológico de los indigenismos permite tener un panorama general sobre cuáles son las lenguas indoamericanas de mayor contacto con el español dentro del espacio geográfico donde se origina el texto, en este caso el otrora Reino de Guatemala, y cómo estas influyen en su español. De acuerdo con los datos que muestra el Diccionario de la Lengua Española, en adelante DLE (2014), y el Diccionario de Guatemala (Armas, 1998), las voces pertenecen a tres distintas lenguas (Cuadro 1).

\begin{tabular}{|l|c|c|c|}
\hline $\begin{array}{l}\text { lengua } \\
\text { originaria* }\end{array}$ & región & $\begin{array}{c}\text { número } \\
\text { de casos }\end{array}$ & voces \\
\hline náhuatl & México & $10(83 \%)$ & $\begin{array}{c}\text { achiote, atole, cacao, camote, chile, } \\
\text { chocolate, güisquil, nixtamal, súchil, jícara }\end{array}$ \\
\hline k'iché & Guatemala & $1(8.5 \%)$ & chay \\
\hline kuna & Panamá-Colombia & $1(8.5 \%)$ & chicha \\
\hline
\end{tabular}

* Las lenguas que se mencionan en este trabajo están registradas de acuerdo con la norma ortográfica vigente en México para escribir nombres de idiomas, en la cual se dicta que deben tener minúscula inicial. De este modo se ha decidido sistematizarlas siguiendo dicha norma e independientemente de lo establecido en sus países de origen.

Cuadro 1: Origen etimológico de los indigenismos presentes en el texto

En el Cuadro 1 se puede observar que diez de las doce voces encontradas pertenecen a la lengua náhuatl, de la familia yuto-azteca, una al k’iché, de la familia mayense, y otra más a la lengua kuna, que forma parte de las lenguas chibchenses.

Pese a que los resultados son esperables, dado su semejanza con los registrados en otros estudios sobre la Guatemala del siglo XVIII, cabe preguntarse por qué, siendo Centroamérica una zona maya, la lengua de mayor influencia léxica es el náhuatl y no el k’iché que, además, cuenta solo con una aparición. De acuerdo con Dakin (2008: 167), existen varios factores que provocan dicha situación: En primer lugar, en la parte sur de Mesoamérica hay colonias donde se habla el náhuat $1^{5}$ (Mathew y Romero 2012), y también es la lengua usada para la comunicación con otros grupos durante el imperio azteca; por último, el náhuatl es la lengua que, junto con el español, se emplea para fines administrativos durante la etapa colonial, considerándose así una de las lenguas generales de la Nueva España (Ricard: 1986), por lo que permite entender la aparición de vocablos de este origen en el texto mencionado. A este respecto, se debe señalar que los datos de la Instruc-

5 Es importante tener presente que el náhuatl es una agrupación lingüística que abarca muchas variantes geográficas y cronológicas, por lo que, aunque se compartieran algunos rasgos estructurales o incluso el propio léxico, no necesariamente existía inteligibilidad entre sí (Dakin 2018). 
ción no constituyen una excepción, pues la localización de mayor número de nahuatlismos dentro de los textos de origen yucateco y centroamericano se ha venido observando desde trabajos anteriores, tales como Polo Cano (2005), Ramírez Quintana (2006), Ramírez Luengo y Quirós García (2015) o Ramírez Luengo, Aquino Melchor y Ramírez Vázquez (2017), entre otros.

Desde el punto de vista cuantitativo, el segundo lugar, a gran distancia del náhuatl, es compartido por el k'iché, y el kuna con una voz cada uno. El k'iché es hablado en las tierras altas centrales de Guatemala, y tiene contacto con el español desde los inicios de la conquista (Romero, 2015), de modo que para el siglo XVIII el español de Guatemala debe de tener basta presencia de indigenismos k'icheanos. Sin embargo, el hecho de que la Instrucción de José Felipe Flores no los muestre posiblemente se deba a que trata temas médicos, donde el náhuatl ya ha llenado los vacíos léxicos que suponen las realidades americanas desconocidas ${ }^{6}$. En este sentido, y de acuerdo con Ramírez Luengo y Quirós García (2015: 201), la escasez de vocablos mayas tiene que ver con circunstancias desfavorecedoras como que "el maya no constituye una de las primeras lenguas de contacto ni tampoco adquiere el papel preponderante en la sociedad colonial que le corresponde al náhuatl [...] pese a ser la lengua de uso habitual" en la región.

Por último, se puede identificar una voz dentro de la Instrucción que pertenece a la lengua kuna. Esta lengua presenta un alto grado de vitalidad actualmente, y se habla en algunas zonas de Panamá y Colombia. El contacto entre dicha lengua y el español es más restringido del que se da con el náhuatl o el k'iché, y se relaciona directamente con la cultura propia de la comunidad (Sherzer 1992). Sin embargo, la presencia de un vocablo de este origen dentro del texto se justifica más por la voz misma que por la lengua en sí, esto se debe a que la palabra chicha se refiere a una "bebida alcohólica usada en América y resultante de la fermentación del maíz, y de otros granos y frutos, en agua azucarada" (DCECH, 1987:194), de modo que la popularidad de la bebida obliga a la incorporación del vocablo al español y explica su posterior expansión por la mayor parte del continente (Buesa Oliver y Enguita Utrilla 1992: 113).

\subsection{Campos semánticos}

A partir de la clasificación de los indigenismos por campos semánticos se puede observar, de manera general, qué tipo de referentes son los que el hablante prefiere incorporar al español por medio de este tipo de voces para nombrar las realidades no existentes en su cultura. En el caso de la Instrucción, de acuerdo con la clasificación establecida en trabajos como Mejías (1980), Polo Cano (2005) y García Frazier (2006), las voces tomadas de las lenguas amerindias se dividen en tres campos: la flora, que incluye la mitad de las entradas léxicas y entre sus voces están aquellas que refieren a la materia prima para preparar alimentos, los alimentos preparados. Y por último el campo de enseres o utensilios, compuesto por instrumentos utilizados ya sea para la curación o para servir los alimentos suministrados durante el cuidado de los inoculados (Cuadro 2).

6 Queda pendiente, para el futuro, revisar otros textos científicos de diferente índole para descubrir si existen mayores aportes del k’iché y, si es así, identificar en qué campos científicos hay mayor aportación léxica por parte de la lengua natural de Guatemala. 


\begin{tabular}{|l|c|c|}
\hline campo léxico & número de casos & voces \\
\hline Alimentos preparados & $4(33.5 \%)$ & atol, chocolate, chicha, nixtamal \\
\hline Flora & $6(50 \%)$ & achiote, camote, cacao, chile, güisquil, súchil \\
\hline Enseres, utensilios & $2(16.5 \%)$ & chay, jícara \\
\hline
\end{tabular}

Cuadro 2: Clasificación por campos semánticos de los indigenismos presentes en el texto

Como se puede apreciar, pese a ser clasificados bajo dos campos semánticos distintos (alimentos preparados y flora), la mayor parte de las voces indígenas presentes en el corpus refieren a realidades relacionadas con la vegetación americana y en la obra, aparecen en el contexto de la dieta a seguir para el cuidado paliativo de la viruela, ya sea natural o inoculada. Este hecho no sorprende, pues desde un punto de vista léxico la alimentación y la medicina siempre han estado estrechamente relacionadas: según Eberenz $(2018,1077)$, las obras medicinales cuentan con numerosas referencias a la comida y bebida, sobre todo en aquellas en las que se busca prevenir o curar alguna enfermedad, que es precisamente la tipología a la que pertenece la Instrucción del médico chiapaneco, más en concreto, y de acuerdo con la clasificación establecida por el mismo autor a los "manuales y compendios generales de medicina que presentan distintas enfermedades y traumatismos, y proponen para cada uno un régimen alimenticio específico, dentro de un conjunto de medicinas terapéuticas", lo que deja en claro el papel de la alimentación, y por ende de los productos de origen vegetal, dentro de la medicina.

En cuanto al campo léxico de los enseres o utensilios, en el corpus se descubren dos voces englobables en él: la primera es jícara, un nahuatlismo muy común que, de acuerdo con Terreros (1787: 388), se trata de "un vaso que comunmente sirve para tomar chocolate", a la que se suma la voz k'icheana chay y, de acuerdo con Batres Jáuregui (1892: 208), “es el nombre que dan al guijarro, ó pedazo de vidrio, que se llama oxidiana, y entre el vulgo, piedra de rayo".

En todos los casos, como se puede ver por el somero análisis que se ha llevado a cabo, los préstamos siempre buscan llenar el vacío léxico que existe en cualquier lengua que ha adoptado un nuevo referente y requiere ser nombrado, cuestión que explica, precisamente, su aparición en un texto perteneciente a una temática como esta, en principio poco adecuada para la presencia de indigenismos.

\subsection{Incorporación de los indigenismos al español del siglo XVIII}

Por lo que se refiere a este punto, es posible sostener la hipótesis de que cada uno de los doce indigenismos presentes en la Instrucción ya están incorporados al español guatemalteco de la época, esto debido a que ninguno de ellos presenta alguna explicación textual o información adicional que aporte al lector el contexto necesario para entender su significado, argumento aportado por diferentes estudiosos para justificar la no integración de estos elementos en un momento específico (Enguita Utrilla 2010). Por supuesto, el empleo de vocablos integrados por parte del doctor chiapaneco no sorprende, sino que responde, primero, al origen del autor, y en segundo lugar al 
hecho de que la intención de Flores sea lograr una amplia difusión del texto en todo el reino de Guatemala para que se pueda llevar a cabo con éxito la inoculación, por lo que necesariamente debe estar utilizando un lenguaje en general y léxico en particular cercano a la coloquialidad dentro del español de esa zona geográfica específica, para que así sea claro para todos.

En concreto, la integración de los indigenismos en el discurso de la Instrucción sobre el modo de practicar la inoculación de las viruelas se comprueba a partir de sus contextos de aparición, que son mostrados en los siguientes ejemplos.

1) Los indios pueden fácilmente substituir sus puntas afiladas de chayes con que acostumbran sangrar. (Flores, 1794: 5)

2) En los pueblos de tierra caliente las verduras del país, como plátano, camote, \&c. y en las tierras frías; manzanas, perotes, y güisquiles, de que se dará su ración a los niños. (Flores, 1794: 5)

3) Es preciso que las tortillas se hagan con más cuidado, que se limpie y se muela bien el nixtamal, para que saliendo blancas, delgadas y suaves, sean más fáciles de digerir. (Flores, 1794: 5)

4) Los alimentos han de ser líquidos de atole, chocolate o caldo, dándolos alternativamente de este modo: por la mañana al desayuno. (Flores, 1794: 5)

5) No coman mucho chile, ni beban chocolate de suchiles. (Flores, 1794: 6)

6) Tomarán una jícara de chicha o de agua dulce bien echa [sic]. (Flores, 1794: 8)

7) Se batirá un poco de chocolate, o masa de cacao, con achiote, que es con lo que los indios hacen el batido. (Flores, 1794: 8)

De este modo, en los ejemplos anteriores es interesante observar que las voces nixtamal, chocolate, cacao y achiote están integradas ya que no solo no presentan la glosa léxica necesaria en los casos de no integración (Enguita Utrilla 2010), sino que además a partir de ellas se explica la composición de otros alimentos preparados, en este caso el batido y las tortillas. Del mismo modo, salta a la vista la composición de referentes a partir de dos voces de origen indígena, jícara de chicha y chocolate de súchil, lo que se puede interpretar como una prueba adicional del natural manejo de este tipo de léxico en la lengua y, por tanto, de su incorporación a la misma.

\subsection{Primeras dataciones generales y centroamericanas}

Las primeras dataciones son el registro escrito más antiguo que se tiene hasta el momento de una voz indígena dentro del español ${ }^{7}$. Este tipo de datos se consigue a partir de la búsqueda de los vocablos en cuestión en corpus del español colonial, y pueden ser generales, es decir, el primer registro absoluto, o de un área geográfica específica. En este caso concreto, se trabajarán las primeras dataciones generales y las primeras dataciones centroamericanas, ya que la Instrucción tiene su origen en esa zona.

Cabe señalar que, al ser tan tardío, finales del siglo XVIII, lo esperable es no encontrar primeras dataciones en el documento escrito por José Felipe Flores, y de hecho en Boyd-Bowman (2015)

7 Aunque es de sobra conocido, conviene señalar que localizar una primera datación no significa que el vocablo en cuestión no estuviera en la lengua oral desde antes, ni tampoco que, conforme avancen las investigaciones léxicas, no pueda aparecer una retrodatación más temprana en otro documento. 
y el Corpus Diacrónico del Español (CORDE) se descubren casos anteriores a esta fecha para: atole (México, 1564-Guatemala 1716), chile (México, 1540-Guatemala, 1548), chocolate (México, 1581-Guatemala, 1582), jícara (México, 1532-Guatemala, 1568), chicha (México, 1532-Guatemala, 1635), cacao (México, 1525-Guatemala, 1550) y achiote (México, 1576-Guatemala, 1588).

Ahora bien, frente a los casos anteriores, las voces chay y güisquil sí presentan su primera datación en la Instrucción, ya que sus registros más antiguos hasta el momento eran de 1949 para el primero y 1933 para el segundo, ambos en Guatemala. Respecto a los términos camote (México, 1551), súchil (México, 1554) y nixtamal (México, 1591), se encuentran sus primeras dataciones absolutas desde el siglo XVI en la ahora Ciudad de México, pero lo cierto es que su aparición dentro de la Instrucción puede ser considerada como primera datación en Centroamérica, lo que aporta un dato de interés acerca de la profundidad histórica de estas voces en el español de la región.

\section{Conclusiones}

Con esta investigación se pretende contribuir a los estudios sobre el léxico médico en el continente americano, ya que actualmente es un área poco explorada, pero con muchos datos que aportar a la configuración léxica del español de América. El caso que aquí se observa es la incorporación de voces indígenas en el ámbito de la medicina, lo que da como resultado la interacción entre el campo de la alimentación y los tratados médicos como la Instrucción sobre el modo de practicar la inoculación de las viruelas del Dr. José Felipe Flores. La influencia de la cocina dentro del quehacer de la medicina es esperable, sobre todo en la tradición indoamericana ${ }^{8}$ que, como ya se mencionó anteriormente, es incorporada por los españoles a la cultura occidental. No obstante, para llevar a cabo esto es necesario integrar también las denominaciones de los productos utilizados, que en muchos casos deberán ser los llamados indigenismos, debido a que se trata de realidades puramente americanas.

Así pues, se puede decir que la presencia de indigenismos en la Instrucción sobre el modo de practicar la inoculación de las viruelas, de una importancia cuantitativa relativa pero cualitativa indudable, se explica por tres razones claras: 1) el origen del autor, que lo familiariza con las voces indígenas ya incorporadas al español de Guatemala en el siglo XVIII; 2) la finalidad divulgativa y no estrictamente científica del texto, pese a ser un texto de índole medicinal, así como el público, no necesariamente especializado, al que va dirigido; y 3) la relación que existe entre los indigenismos y el campo léxico de la alimentación, ya que el empleo de comida endémica de la zona complica la presencia de términos equivalentes en el español, muchas veces inexistentes, lo que lleva al médico chiapaneco a incorporar específicamente el léxico de origen amerindio que nombra dichas realidades.

En cuanto a los orígenes etimológicos de las voces indígenas que se registran en el texto, estas pertenecen a tres lenguas distintas, náhuatl, k’iché y kuna, pero con una fuerte tendencia al predominio del náhuatl pese a ser una zona maya, algo que se explica debido a que el maya no es una

8 Estas prácticas se han registrado desde documentos muy tempranos como en el Códice de la Cruz-Badiano que "es un recetario con los tratamientos recomendados por Martín de la Cruz, médico indígena encargado del cuidado de los niños internos del Colegio de Santa Cruz de Tlatelolco para contrarrestar las principales enfermedades que afectaban a los indígenas mexicanos a mediados del siglo XVI" (Viesca y Ramos 2012: 74). 
de las lenguas generales del periodo colonial en Mesoamérica, mientras que el náhuatl sí adquiere este papel. Respecto a los campos semánticos, como ya se dijo, todos los vocablos guardan una relación más o menos directa con la cocina, y se distribuyen ente alimentos preparados, flora y enseres o utensilios; mientras que las enfermedades y sus manifestaciones, por ejemplo, siguen siendo nombradas con voces patrimoniales o adaptaciones de ellas, lo que demuestra que este factor es de gran relevancia a la hora de entender los resultados de un análisis como este.

Por último, y en relación con el grado de integración de estas voces y su cronología, es importante mencionar que, considerando los criterios utilizados por Enguita Utrilla (2010: 202) a este respecto, cada uno de los indigenismos localizados se encuentran integrados en el español dieciochesco de Guatemala, y que algunas voces aparentan tener su primera datación en el corpus analizado, como es el caso de chay y güisquil de forma general y camote, nixtamal y súchil para el caso de Centroamérica.

Naturalmente, un análisis puntual como este no resuelve todas las dudas existentes al respecto, por ejemplo, quedan pendientes temas por explorar que salen de los límites de este trabajo como la toponimia o la búsqueda de voces indígenas en textos científicos de otra índole para observar su comportamiento, todo lo cual sin duda enriquecerá el conocimiento que por el momento se posee acerca de la historia del léxico americano.

\section{Referencias bibliográficas}

Armas, D. (1998). Diccionario de la expresión popular guatemalteca. Guatemala: Piedra Santa.

Aznar López, J. (1998). José Felipe Flores, científico chiapaneco de la Ilustración hispanoamericana. Tuxtla Gutiérrez: Coneculta Chiapas.

Barona Vilar, J. L. (2019). José Felipe Flores. In Real Academia de la Historia (org.), Diccionario biográfico electrónico. <http://dbe.rah.es/ biografias/1936[9/jose-felipe-flores>

Batres Jáuregui, A. (1892). Vicios del lenguaje y provincialismos de Guatemala. Ciudad de Guatemala: Tipografía Nacional.

Buesa Oliver, T.; \& Enguita Utrilla, J. M. (1992). Léxico del español de América: Su elemento patrimonial e indigena. Madrid: Editorial Mapfre.

[CORDE]: Real Academia Española (2019). Banco de datos CORDE. Corpus diacrónico del español. <http:// www.rae.es>

Dakin, K. (2008). El náhuatl de las memorias: Los rasgos de una lingua franca indígena. In J. L. de Rojas (Ed.), La Etnohistoria de América: Los indígenas, protagonistas de su historia (pp. 167-189). Buenos Aires: Paradigma Indicial.

[DCECH] Corominas, J. \& Pascual J. A. (1980-1991). Diccionario crítico etimológico castellano e hispánico. Madrid: Gredos.

[DLE]: Real Academia Española (2014). Diccionario de la Lengua Española. Madrid: Espasa-Calpe.

Eberenz, R. (2018). Discurso y léxico de la alimentación en los tratados médicos medievales y renacentistas. In M. L. Arnal Purroy (Ed.), Actas del X Congreso Internacional de Historia de la Lengua Española. Zaragoza, 7-11 de septiembre de 2015 (vol. 2) (pp. 1075-1088). Zaragoza: Institución Fernando el Católico, Excma, Diputación Provincial de Zaragoza. 
Enguita Utrilla, J. M. (2010). Indoamericanismos léxicos y estructuras discursivas en la Relación de Cristobal de Molina. In C. de Molina, P. Jiménez del Campo, P. Cuenca Muñoz, \& E. López Parada (Eds.), Relación de las fábulas y ritos de los incas (pp. 199-216). Madrid: Iberoamericana, Vervuert.

Flores, J. F. (1794). Instruccion sobre el modo de practicar la inoculacion de las viruelas, y metodo para curar esta enfermedad, acomodado a la naturaleza, y modo de vvir de los indios, del reyno de Guatemala. Guatemala: Oficina de Don Ignacio Beteta.

García Frazier, E. (2006). Préstamos del náhuatl al español mexicano. Hesperia: Anuario de filología hispánica, 9, 75-86.

Garzón y Rincón, A. F. (2014). El notable caso del Dr. José Felipe Flores. Salud en Chiapas, 1, 22-28.

Gómez de Enterría, J. (2012a). El vocabulario médico de los novatores en el siglo XVIII. In M. T. García Godoy (Ed.), El español del siglo XVIII. Cambios diacrónicos en el primer español moderno (pp. 55-81). Berna: Peter Lang.

. (2012b). El vocabulario de la medicina del siglo XVIII a través de las traducciones de los hermanos Juan y Félix Galisteo Xiorro. In C. Montero (Ed.), Actas del VIII Congreso Internacional de Historia de la Lengua Española, II (pp. 1405-1422). Santiago de Compostela: Meubook.

- (2013). Higiene y salud en las traducciones médicas del francés al español durante el siglo XVIII. Panace@. Revista de medicina, lenguaje y traducción, 14, 38, 287-295.

- (2014). El vocabulario de las fiebres epidémicas en el español del siglo XVIII. España y México. In José Luis Ramírez Luengo \& E. P. Velásquez Upegui (Eds.), La historia del español hoy. Estudios y perspectivas (pp. 199-216). Lugo: Editorial Axac.

Gómez de Enterría, J.; \& Gallardo, N. (2010). Las versiones de medicina y botánica y la nueva terminología científica en el siglo XVIII. Cuadernos del Instituto de Historia de la Lengua, 4, 55-75.

Gutiérrez Rodilla, B. M. (1993). Los términos relacionados con la medicina en el Diccionario de Autoridades. Boletín de la Real Academia Española, 73, 260, 463-512.

- (1994). El vocabulario de las fiebres epidémicas en español del siglo XVIII. Revista de Lexicografía, 1, 149-162.

. (1996). El léxico de la medicina en el diccionario de Esteban Terreros y Pando. En A. Alonso González (Ed.), Actas del III Congreso Internacional de Historia de la Lengua Española: Vol. II (pp. 1327-1342). Arco Libros.

[LEXHISP]: Boyd-Bowman, P. (2003). Léxico hispanoamericano. 1493-1993. Nueva York: Hispanic Seminary of Medieval Studies. $<$ http://textred.spanport.lss.wisc.edu>

Matthew, L. E.; \& Romero, S. (2012). Nahuatl and Pipil in Colonial Guatemala: A Central American Counterpoint. Ethnohistory, 54, 4, 765-783.

Mejias, H. A. (1980). Préstamos de las lenguas indígenas en el español americano del siglo XVII. México: Universidad Nacional Autónoma de México.

Parcero Torre, C. (2011). Los pareceres médicos en un texto del siglo XVIII colombiano. Cuadernos de la ALFAL, 2, 196-203.

Polo Cano, N. (2005). Algunos indigenismos léxicos en el español de Guatemala del siglo XVIII. Res Diachronicae, 4, 185-202.

Quirós García, M.; \& Ramírez Luengo, J. L. (2015). Observaciones sobre el léxico del español de Yucatán (1650-1800). Revista de Filología Española, 95, 1, 183-210.

Ramírez Luengo, J. L. (2014). Un aporte a la historia del léxico médico en América: El vocabulario de la viruela en la Nueva España dieciochesca. Anuario de Letras. Lingüística y Filología, 2, 1, 181-214. 
. (2015). Algunas notas sobre el léxico médico en la Nueva España dieciochesca: Voces cultas y populares en la denominación de las enfermedades. Cuadernos Dieciochistas, 16, 291-310.

. (2019). América y la medicina: Los indigenismos en el Florilegio medicinal de Juan de Esteyneffer (1712). Nuevas Glosas. Estudios de Literatura y Lingüística, 1, 265-268.

. (En prensa). El léxico de la viruela en Guatemala del siglo XVIII: Algunas notas sobre la Instrucción sobre el modo de practicar la inoculación de las viruelas de José Felipe Flores (Ciudad de Guatemala, 1794).

Ramírez Luengo, J. L.; Aquino Melchor, S. A.; \& Ramírez Vázquez, J. J. (2017). Indigenismos léxicos en el español guatemalteco del siglo XVIII. Lengua y habla, 21, 111-125.

Ramírez Quintana, P. A. (2006). Caracterizadores léxicos del español colonial en Campeche. In C. Company Company, \& J. G. Moreno de Alba (Eds.), Actas del VII Congreso Internacional de Historia de la Lengua Española (1471-1484). Madrid: Arco/Libros.

Ricard, R. (1986). La Conquista Espiritual de México. México: Fondo de Cultura Económca.

Romero, S. (2015). Language and ethnicity among the K'ichee' Maya. University of Utah Press.

San Martín Gómez, J. A. (2019). Aproximación al indigenismo léxico en el español de El Salvador (siglos XVII-XVIII). RILEX. Revista sobre investigaciones léxicas, 2, 2, 121-134.

Sherzer, J. (1992). Formas del habla kuna: Una perspectiva etnográfica. Quito: Ediciones Abya Yala.

Terreros, E. de (1987 [1786]). Diccionario castellano con las voces de ciencias y artes y sus correspondientes en als tres lenguas francesa, latina e italiana. Madrid: Arco/Libros.

Viesca Treviño, C.; \& Ramos de Viesca, M. (2012). Códice de la Cruz-Badiano. La colección de códices de la Biblioteca Nacional de Antropología e Historia. Revista de Arqueología Mexicana, 42 (edición especial), $74-76$. 
